\title{
Acesso cirúrgico palatino para tracionamento ortodôntico de canino
}

\author{
Patatal surgical access for canine orthodontic traction: a case study
}

Acceso quirúrgico palatino para la tracción de ortodoncia canina: un estudio de caso

Lucas Menezes dos Anjos ${ }^{1}$, Aurélio de Oliveira Rocha ${ }^{2 *}$, Rafaela de Menezes dos Anjos Santos ${ }^{1}$, Maria de Nazaré Oliveira Rocha ${ }^{3}$, Nailson Silva Meneses Júnior ${ }^{1}$, Mellany Cristie Ramos Barros ${ }^{1}$, Thaine Oliveira Lima², Ingrid de Melo Silva², Denílson Oliveira Correia da Silva², Maraiza Alves de Oliveira'.

\section{RESUMO}

Objetivo: Descrever um caso de impactação de canino superior direito (unidade 13) em que foi realizado acesso cirúrgico para posterior tracionamento ortodôntico e restabelecimento da oclusão. Detalhamento de caso: Paciente 19 anos de idade, sexo feminino, em tratamento ortodôntico, foi encaminhada pelo ortodontista devido presença de espaço entre a unidade dentária 14 e unidade 12 . Clinicamente notou-se ausência do elemento 13 , e em radiografia periapical constatou-se impactação dentária. A tomografia evidenciou impactação com giroversão e trajetória do elemento 13 situada na região palatina, sem dilaceração radicular. Os caninos são elementos fundamentais para o equilíbrio funcional do sistema estomatognático, assim como para estética. Estas unidades dentárias representam a segunda maior frequência de impactação, ficando atrás somente dos terceiros molares inferiores. Após alinhamento dos dentes por meio de aparelho ortodôntico do tipo autoligante, foi sugerido realização de acesso cirúrgico para tracionamento e desimpactação da referida unidade. Considerações finais: A realização da cirúrgia para tracionamento das unidades impactadas é segura, funcional e com baixo custo. Dispor de exames imagiológicos, como tomografia computadorizada, é de extrema importância para o correto planejamento e melhor prognóstico.

Palavras-chave: Dente impactado, Ortodontia corretiva, Cirurgia.

\begin{abstract}
Objective: To describe and discuss a case study of upper right canine impaction (unit 13) in which surgical access was performed for later orthodontic traction and restoration of occlusion. Case details: A 19-year-old female patient undergoing orthodontic treatment was referred by the orthodontist due to the presence of a space between dental unit 14 and unit 12. Clinically, the absence of element 13 was noted, and on periapical radiography it was found dental impaction. The tomography showed impaction with gyroversion and trajectory of element 13 located in the palatal region, without root laceration. Permanent canines, fundamental elements to the functional and aesthetic balance of the stomatognathic system, represent the second highest frequency of impaction, which can result in complications such as: migration of adjacent teeth, root resorption, dentigerous cysts, loss of arch length, greater susceptibility to infections and referred pain. After aligning the teeth using a self-ligating orthodontic appliance, surgical access was suggested for traction and disimpaction of the referred unit. Final considerations: The surgery for traction of the impacted units is safe, functional and inexpensive. Having imaging tests, such as computed tomography, is extremely important for correct planning and better prognosis.
\end{abstract}

Keywords: Impacted tooth, Corrective orthodontics, Surgery.

\section{RESUMEN}

Objetivo: Describir y discutir un estudio de caso de impactación del canino superior derecho (unidad 13) en el que se realizó un acceso quirúrgico para posterior tracción ortodóncica y restauración de la oclusión. Detalles del caso: Una paciente de 19 años en tratamiento de ortodoncia fue referida por el ortodoncista debido a la presencia de un espacio entre la unidad dental 14 y la unidad 12. Clínicamente se notó la ausencia

${ }^{1}$ Universidade Federal de Sergipe (UFS), Aracaju - SE.

2 Universidade Tiradentes (UNIT), Aracaju - SE. *E-mail: aureliorocha2015@gmail.com

${ }^{3}$ Faculdade Maurício de Nassau (UNINASSAU), Arapiraca - AL. 
del elemento 13, y en la radiografía periapical fue encontró impactación dental. La tomografía mostró impactación con giroversión y trayecto del elemento 13 ubicado en región palatina, sin laceración radicular. Los caninos permanentes, elementos fundamentales para el equilibrio funcional y estético del sistema estomatognático, representan la segunda frecuencia más alta de impactación, lo que puede resultar en complicaciones como: migración de dientes adyacentes, reabsorción radicular, quistes dentígeros, pérdida de longitud de arco, mayor susceptibilidad a infecciones y dolores referidos. Luego de alinear los dientes con un aparato de ortodoncia de autoligado, se sugirió el acceso quirúrgico para la tracción y desimpactación de la unidad referida. Consideraciones finales: La cirugía de tracción de las unidades impactadas es segura, funcional y económica. Tener pruebas de imagen, como la tomografía computarizada, es extremadamente importante para una planificación correcta y un mejor pronóstico.

Palabras clave: Diente impactado, Ortodoncia correctiva, Cirugía.

\section{INTRODUÇÃO}

A errupção dentária é cacacterizada pela trajetória fisiológica que os dentes realizam no interior dos ossos maxilares em direção ao plano funcional na cavidade bucal culminado em uma sequência favorável de irrupção (ALMEIDA RR, et al., 2001; MARCELINO VCS, et al., 2017; CAPPELLETTE M, et al., 2008; CARVALHO AAB, et al., 2017). Um dente é considerado incluso quando o mesmo não se acomoda na arcada dentária, continuando parcialmente ou totalmente no interior do osso alveolar, e se a inclusão for criada por uma barreira mecânica, como dentes adjacentes, cistos e tumores benignos, é denominado dente impactado (LIMA ILA, et al., 2009; MARTINS MM, et al., 2009; RIBEIRO ET, et al.,2015).

A impactação ou retenção dos dentes pode ocorrer por diversos fatores, sendo os mais comuns: hereditariedade, retenção prolongada do canino decíduo, presença de cistos, tumores ou supranumerários na região (PRIMO BT, et al., 2011; ALVES EP, et al., 2014; VIEIRA FM, et al., 2014). Cerca de 23,5\% das amostras selecionadas previamente para tratamento ortodôntico apresentam caninos permanentes impactados, sendo essas unidades dentárias as segundas com maior fequência de não erupção (CARDOSO MA, et al., 2011). O osso palatino é a principal região em que ocorre retenção de canino, sendo mais comum no sexo feminino (BISHARA SE, 1992; CRUVINEL MOB, 2018).

Diante de um dente impactado, a decisão de extrair ou mantê-lo utilizando manobras ortocirúrgicas deve passar por uma minuciosa avalição e planejamento, e situações como angulação, espaço disponível no arco para reposicioná-lo e proximidade com a raiz dos incisivos laterais subsequente, devem ser levadas em consideração (FERGUSON JW e PITT SKJ, 2004; FLEMING PS, et al., 2009).

A idade do paciente também deve ser analisada, já que atrelado a isso os tratamentos podem se tornar mais longos e procedimentos mais conservadores como sua manutenção e acompanhamento radiográfico, podem ser considerados para atenuar a morbidade, sendo imprescindível a decisão do paciente sobre a conduta a ser adotada (JUVVADI S, et al., 2012).

Segundo Simão MT, et al. (2012) várias são as possibilidades terapêuticas frente a um caso de impactação de canino, destacando-se: aguardar a erupção espontânea sendo feitas tomadas radiográficas para analisar sua possível movimentação; transplante autógeno, método que consiste em procedimento exclusivamente cirúrgico onde é realizada a extração do canino retido seguido de posterior transplante para um alvéolo artificial realizado no osso alveolar; extração do canino impactado; e exposição cirúrgica para tracionamento ortodôntico.

A utilização de exames de imagem é imprescindível para avaliação e planejamento em casos de impactação dentária (GONDIM CR, et al., 2010). A tomografia computadorizada de feixe cônico (Cone Beam) é considerada um excelente exame de imagem para avaliar dentes impactados, pois permite fazer de forma mais precisa o diagnóstico das anomalias de posição já que seus vários planos de cortes e as decorrentes reconstruções em imagem 3D, passíveis de observação em praticamente todos os ângulos, permitem que o profissional planeje o tracionamento ortodôntico de caninos superiores com maior segurança, o que possibilita avaliar o folículo pericoronário, a região cervical e os dentes vizinhos a partir de um detalhado planejamento, diminuindo os riscos de intercorrências (CONSOLARO A, 2010; GONDIM CR, et al., 2010; WALKER L, et al., 2005). 
Dentre as alternativas terapêuticas, a combinação do tratamento cirúrgico-ortodôntico permite a colagem de um acessório para a tração do canino retido, realizado por meio de acesso cirúrgico à unidade impactada, o que permitirá o tracionamento da unidade em questão e posterior posicionamento na arcada dental (CARDOSO MA, et al., 2011; MARCELINO VCS, et al., 2017).

Diante do exposto, o presente estudo visa descrever uma abordagem conservadora para tratamento de canino permanente superior impactado através do acesso cirúrgico palatino para tracionamento e restabelecimento funcional e estético do sistema estomatognático.

\section{DETALHAMENTO DO CASO}

Paciente LO, 19 anos de idade, gênero feminino, ASA I, em tratamento ortodôntico, foi encaminhada pelo ortodontista devido presença de espaço entre o incisivo lateral superior direto e primeiro pré-molar superior direito (Figura 1). No exame clínico percebeu-se ausência da unidade 13 e leve proeminência na região anterior direta do palato. Após realização de radiografia periapical por meio da técnica de Clark, foi então identificada presença da unidade 13 em condição de impactação, giroversão para mesial e situada na região palatina.

Figura 1- Vista oclusal da maxila.

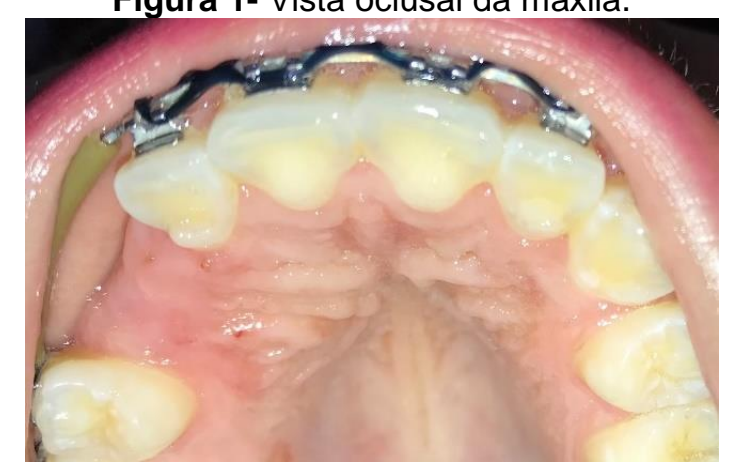

Fonte: Anjos LM, et al., 2021.

Com o advento da tomografia computadorizada de feixe cônico, por meio de analise dos cortes coronais, axiais e sagitais, evidenciou-se presença da unidade 13 situada no osso palatino, em relação de impactação com a raiz da unidade 12, giroversão para mesial, sem dilaceração radicular, presença de saco pericoronário, formação radicular completa não possuindo, portanto, força eruptiva neste elemento, e fenda óssea acima de sua coroa (Figura 2).

Figura 2- Tomografia computadorizada.

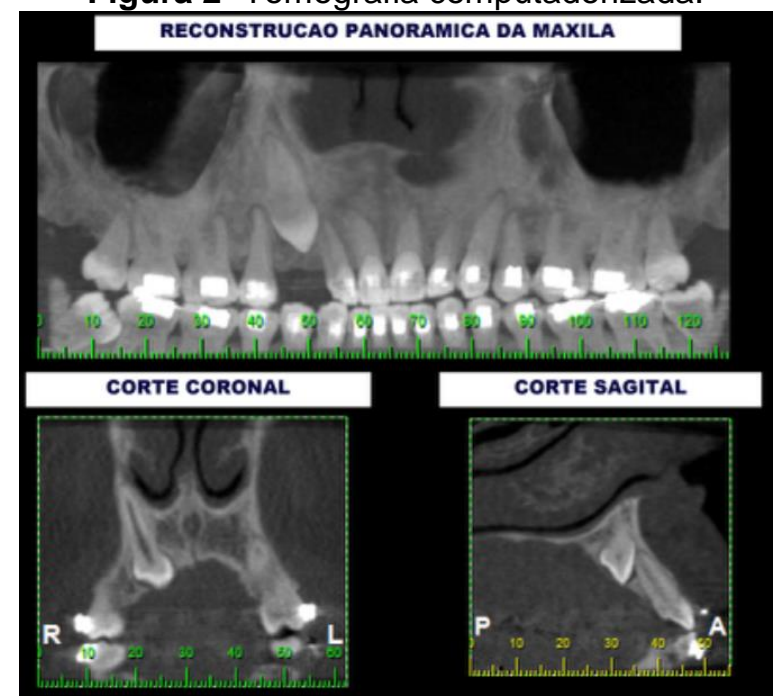

Fonte: Anjos LM, et al., 2021. 
A proposta de tratamento para o caso foi exposição cirúrgica do elemento dentário 13 por meio de retalho palatino associado a colagem de bracket e posterior tracionamento com o advento de aparelho ortodôntico do tipo autoligante.

Seguindo os protocolos de antissepsia e assepsia, foi feita anestesia tópica com benzocaína $200 \mathrm{mg} / \mathrm{g}$ (DFL $\AA$ ), seguida de bloqueio do nervo alveolar superior médio direito, bloqueio do nervo palatino marior e bloqueio do nervo nasopalatino com lidocaína 2\% com epinefrina 1:100.000 (DFL®), assim como anestesia infiltrativa com a finalidade de diminuir o sangramento regional possibilitando um campo de trabalho mais limpo e de melhor visualização. Após certificar-se da analgesia da região, foi feita incisão intrassucular palatina com bisturi número 15-C (SOLIDOR®) estendendo-se da mesial da unidade 16 a distal da unidade 23.

Segue-se então com o descolamento subperiosteal da mucosa palatina buscando campo de visão claro e seguro. Uma broca carbide esférica PM número 5 (JET®), acoplada em motor de alta rotação, foi utilizada para expor a coroa da unidade dentária em questão e permitir colagem do bracket ortodôntico. $O$ desgaste ósseo deve ser seletivo e conservador respeitando estruturas vizinhas como as raízes do incisivo central e lateral direitos (Figura 3).

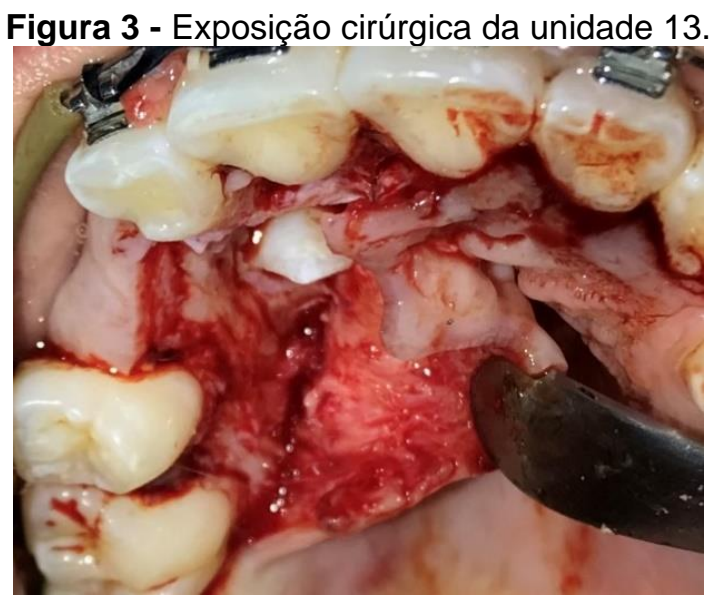

Fonte: Anjos LM, et al., 2021.

Após exposição da coroa da unidade 13, foi seguido o protocolo de colagem do bracket de aço (MORELLI ${ }^{\circledR}$ ), o qual consiste em condicionamento ácido do esmalte dental com ácido fosfórico 37\% (FGM®) por 30 segundos, lavagem abundante do ácido com soro fisiológico estéril, controle de umidade da coroa, seguida de aplicação de adesivo ambar APS (FGM®) e fotopolimerização (SDI®) por 20 segundos (Figura 4). A aplicação do ácido fosfórico deve ser seletiva ao esmalte dentário impedindo seu extravasamento para os tecidos moles, conduta adotada para evitar queimadura dos tecidos, o que aumentaria as chances de necrose tecidual no pós-operatório.

Figura 4 - Aplicação de ácido fosfórico.

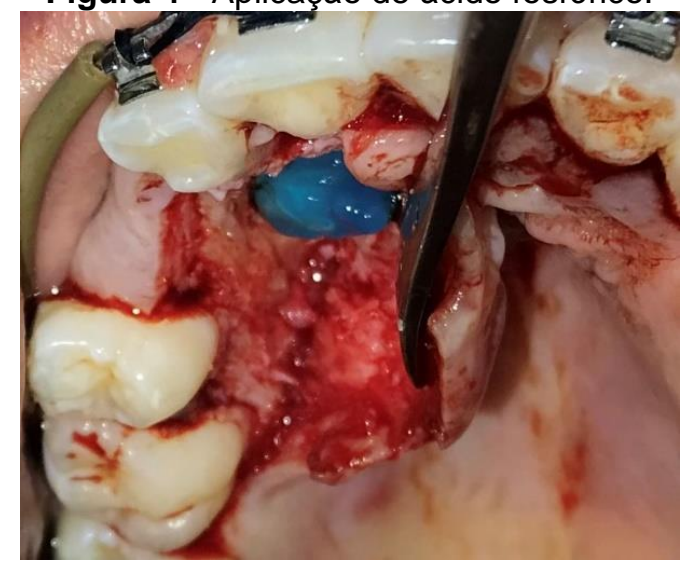

Fonte: Anjos LM, et al., 2021. 
Um fio ortodôntico duro elástico CRNI redonto $0,30 \mathrm{~mm}$ (MORELLI®) foi entrelaçado para garantir maior resistência a tração e fixado no bracket. Com o substrato devidamente preparado por meio do protocolo adesivo, o acessório ortodôntico especifico para canino superior foi então colado a coroa da unidade 13 por meio de resina composta (BIODINÂMICA®) (Figura 5). Após a polimerização da resina composta, foi realizada força de tração manual no fio ortodôntico para certificar-se que a adesão do conjunto bracket-resinacoroa tinha sido satisfatória.

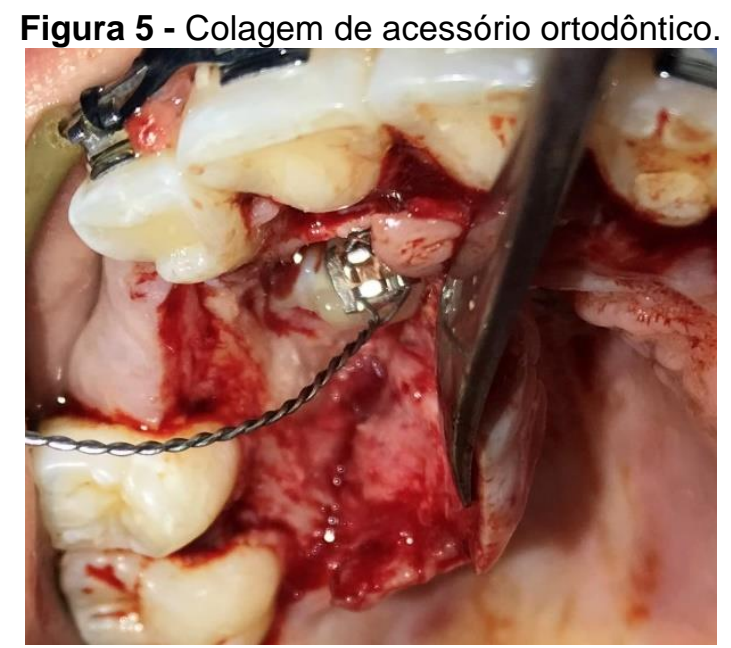

Fonte: Anjos LM, et al., 2021.

Após limpeza do campo cirúrgico com irrigação copiosa com soro fisiológico estéril, a mucosa palatina foi adaptada e repousada sobre o osso palatino e suturada com fio de nylon 3-0 (SHALON®) por meio de sutura do tipo suspensória (Figura 6). Para controle da dor e inflamação pós-operatória foi prescrito Nimesulida $(100 \mathrm{mg})$ de $12 / 12$ horas por três dias, Dipirona $(500 \mathrm{mg})$ de $04 / 04$ horas por três dias e Clorexidina $(0,12 \%)$ sendo solicitado bochecho $12 / 12$ horas dois dias depois do procedimento cirúrgico.

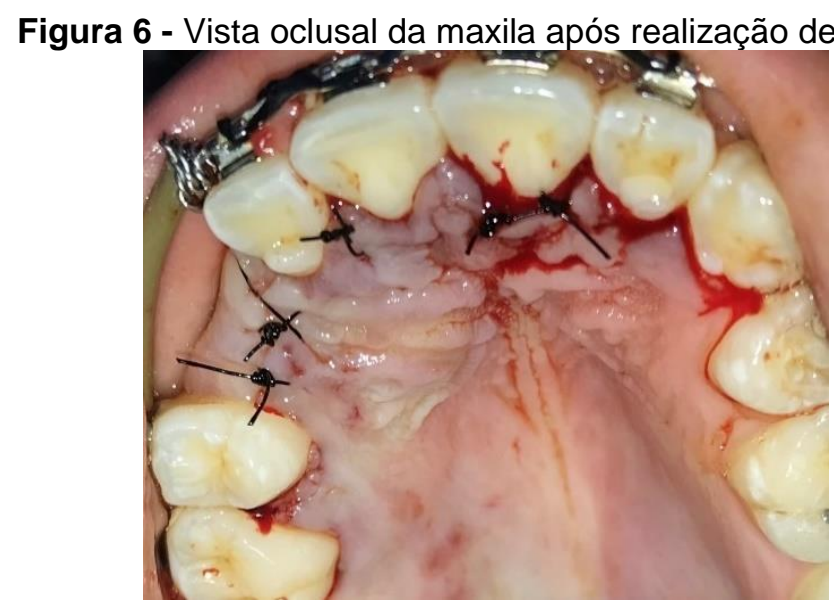

Fonte: Anjos LM, et al., 2021.

\section{DISCUSSÃO}

Os caninos superiores permanentes têm a segunda maior incidência de impactação, ficando atrás somente dos terceiros molares, podendo ocorrer uni ou bilateralmente (BROWN NL e SANDY JR, 2001). Segundo Kanavakis G, et al. (2015), tem maior prevalência no sexo feminino, representando $61 \%$ dos casos em comparação ao sexo masculino (39\%), estando relacionados em sua grande maioria na região do palato numa proporção de 2:1 em relação à posição labial. No caso clínico em questão, tanto a prevalência no sexo feminino como sua ocorrência no palato, reforçam os dados apontados na literatura.

A impactação dos caninos permanentes por palatina pode ter origem multifatorial, incluindo fatores de ordem genética, distúrbios endócrinos, retenção prolongada ou perda prematura do elemento decíduo, 
posição ectópica do germe dentário, presença de fenda palatina ou alveolar, anquilose, presença de cistos, dilaceração radicular, agenesia do incisivo lateral, trajeto longo e tortuoso de erupção e presença de dente supranumerário na região de erupção do canino (VIEIRA FM, et al., 2014). Após anamnese detalhada, visando identificar o motivo da impactação no caso clínico, identificou-se posição ectópica do germe dentário com giroversão para mesial.

De acordo com Duncam WR, et al. (1983), a remoção de interferências mecânicas como extranumerários, patologias e até recuperação de espaço pode ser uma alternativa quando se visa aguardar a irrupção espontânea do canino retido. Mas Segundo Jacoby H, (1983), caninos por palatino raramente irrompem espontaneamente por vestibular, e quando o fazem podem irromper em posição ectópica.

Caso seja planejado o acompanhamento para possível irrupção espontânea é importante que se faça um controle radiográfico periódico para avaliar alterações patológicas (TORMENA JR, et al., 2004). Com o advento de aparelho ortodôntico do tipo autoligante, foi feito recuperação do espaço destinado a unidade 13 visando a irrupção espontânea, após um período de três meses, percebeu-se que não ouve nenhum tipo de movimentação da unidade e que a mesma se encontrava no palato.

Diante das várias opções de tratamento para caninos impactados, tem-se optado pela conduta ortocirúrgica, pelo fato de ser um tratamento mais conservador e menos arriscado (FRANCO AA, et al., 2006). Segundo Giglio TJ e Grugrl JA (2010) não existe um protocolo definitivo sobre o melhor tratamento, sendo este baseado em um planejamento de caráter multidisciplinar, como Cirurgia, Ortodontia, Periodontia e Radiologia, baseado em exames clínicos e radiográficos, além da preferência ou experiência dos profissionais envolvidos com as técnicas e materiais disponíveis.

Franco AA, et al. (2006) e Manzi FR, et al. (2011) salientaram a importância do tracionamento dos caninos sempre que possível, em decorrência do imprescindível valor morfofuncional e estético, sendo conveniente nos movimentos de lateralidade, estabelecido como um membro de proteção do sistema estomatognático. Ao optar-se pelo tracionamento como medida terapêutica, o prognóstico desta conduta deve ser definido e esclarecido previamente ao paciente, assim como seus riscos potenciais, como a possibilidade de ocorrência de necrose do incisivo adjacente, anquilose, reabsorção do canino e dentes adjacentes e perda do tecido de sustentação (PROFFIT WR, 1993; SILVA K, el al., 2019).

Segundo Dias SD, et al. (2020), em paceintes com idade entre 13 e 19 anos, o tracaionamento ortodôntico por meio de exposição cirúrgica apresenta prognóstico favorável. Já em pacientes com idade superior a 25 anos, como trás Proffit WR (1993), movimentação ortodôntica de unidades dentárias impactadas corre um alto risco de sofrer anquilose. O caso em estudo, por dispor de um paciente de 19 anos, idade que a literatura indica como favorável para realização do tratamento ortocirúrgico, optou-se por realizar o tracionamento.

Os exames radiográficos são utilizados para diagnóstico, prognóstico e plano de tratamento das inclusões dentárias, sendo eles os métodos radiográficos: de Clark para localização vestíbulo-palatal; radiografias oclusais para determinar a relação dos caninos com a linha media; radiografia panorâmica para avaliação geral; telerradiografia lateral para avaliação da posição anteroposterior do dente deslocado, a inclinação e localização vertical no alvéolo e a relação com estruturas faciais como seio maxilar e base nasal; e a tomografia computadorizada (LANDIM FS, et al., 2010). A técnica de Clark e a tomografia computadorizada foram os exames de imagens utilizados no relato do caso descrito.

De acordo com Capellete $\mathrm{M}$, et al. (2008), a tomografia computadorizada é o exame imaginológico que permite identificar com maior precisão a relação do canino impactado com as raízes do incisivo leteral permanente, além de definir a real extensão de uma possível reabsorção, ocorrência de anquilose e dilaceração. A alta incidência de reabsorção das raízes dos incisivos laterais pela impactação dos caninos faz necessário que a relação dessas duas unidades dentárias seja analisada de maneira criteriosa (LANDIM FS, et al., 2010; DIAS SD, 2020).

Ericson S e Kurol J (1987) verificaram que em $60 \%$ dos casos de reabsorções dos incisivos laterais pelos caninos impactados, a extensão destas reabsorções era efetivamente identificada com o advento das tomografias computadorizadas. Diante do exposto, a tomografia computadorizada foi utilizada para 
visualização e maior acurácia sobre a posição da unidade dentária impactada e sua relação com as raízes da unidade 12. O exame evidenciou a impactação da coroa da unidade 13 no ápice radicular da unidade 12 , mas sem presença de reabsorção.

A literatura mostra que a taxa de irrupção espontânea de caninos impactados no osso palatino é muito baixa, sendo o tracionamento ortodôntico por meio da exposição cirúrgica uma alternativa segura e com excelentes resultados na literatura (CASTRO RRSML 2020; DIAS SD 2020; FILHO CL, et al., 2011; SIMÃO MT, et al., 2012). Este procedimento consiste em acessar o canino impactado para a fixação do acessório ortodôntico e utilizar a mecânica até seu posicionamento no arco dentário (SILVA K, et al., 2019). Tais achados na literatura serviram de base para decisão do tratamento proposto no caso em estudo.

Segundo Tanaka O, et al. (2000), a monobra cirúrgica em que é realizada perfuração na coroa do canino impactado é feita nos casos que há difuculdade de perfeita colagem do acessório ortodôntico na coroa da referente unidade dentária. Porém, segundo Simão MT et al. (2012), os possíveis danos pulpares que podem ocorrer ao realizar essa técnia pela dificuldade de acesso ideal, a torna pouco usual.

Silva Filho OG, et al. (1994) dizem que nos casos em que o dente impactado encontra-se muito alto em relação ao plano oclusal e a colagem insatisfatória poderia culminar em descolamento do acessório ortodôntico, a perfuração da coroa no sentido vestíbulo palatino é indicada. Para o caso em questão foi feita exposição cirúrgica seguida de colagem do acessório ortodôntico em esmalte por meio de sistema adesivo e resina composta, como sugere Franco AA, et al. (2006) por ser um tratamento mais conservador e menos arriscado.

A decisão adotada pelo profissional deve envolver um planejamento adequado do caso, sendo principalmente avaliada a posição do canino impactado e sua angulação em relação à linha média, assim como a adesão do paciente ao tratamento e as possíveis desvantagens da técnica (CASTRO RRSML, et al., 2020; PROFFIT WR, 1993; SILVA K, et al., 2019). O cirurgião oral e o ortodontista devem estar em concordância sobre a melhor conduta adotada, trabalhando de forma multidisciplinar com periodontista e radiologista, avaliando a localização tridimensional do dente e determinando a sua viabilidade, afim de favorecer o prognóstico e satisfazer a expectativa do paciente.

A impactação de unidades dentais gera problemas funicionais ao sistema estomatognático, assim como problemas de autoestima para o paciente, principalmente quando associado a dentes anteriores. A realização de acesso cirúrgico para posterior tracionamneto orotodôntico é um tratamento seguro, funcional e de baixo custo. Dispor de exames imaginológicos para o correto planejamento do caso, execução de uma técnica cirúrgica meticulosa e uma planejamento multidisciplinar são peças fundamentais para alcançar resultrados previsíveis e que tragam melhor qualidade de vida para o paciente.

\section{REFERÊNCIAS}

1. ALMEIDA RR, et al. Abordagem da impactação e/ou irrupção ectópica dos caninos permanentes: considerações gerais, diagnóstico e terapêutica. Revista Dental Press de Ortodontia e Ortopedia Facial, 2001; 6(1): 93-116.

2. ALVES EP, et al. Prevalência e posição de caninos superiores impactados e sua relação com reabsorção radicular. Revista da Faculdade de Odontologia da UPF, 2014; 19(2): 180-184

3. BISHARA SE. Impacted maxillary canines: a review. American Journal of Orthodontics, 1992; 101(2): $159-171$.

4. BISHARA, SE. et al. Management of impacted canines. J. Orthod, 1976, 69: 371-387.

5. BROWN NL e SANDY JR. Spontaneous improvement in posicion of canines from apparently hopeless position. Int $J$ Paediatr Dent, 2001; 1(1):64-68.

6. CAPPELLETTE M, et al. Caninos permanentes retidos por palatino: diagnóstico e terapêutica: uma sugestão técnica de tratamento. Revista Dental Press de Ortodontia e Ortopedia Facial, 2008; 13(1): 60-73.

7. CARDOSO MA, et al. Tracionamento de canino permanente superior: relato de caso clínico. Revista Clínica de Ortodontia Dental Press, 2011; 10(4): 108-121.

8. CARVALHO AAB, et al. Importância da tomografia computadorizada de feixe cônico na avaliação de canino incluso na maxila. Revista Brasileira de Odontologia, 2017; 74(2): 143-149.

9. CASTRO RRSML, et al. Critérios para decisão do tratamento de caninos inclusos: Exodontia versus Tracionamento. Brazilian Journal of health Review, 2020; 3(6): e 15872-15878.

10. CONSOLARO A. Tracionamento ortodôntico: possíveis consequências nos caninos superiores e dentes adjacentes Parte II: reabsorção cervical externa nos caninos tracionados. Dental Press J Orthod, 2010; 15(5):23-30. 
11. CRUVINEL MOB, et al. Utilização de cantilever para tracionamento de canino impactado. OrtodontiaSPO, 2018; 51(1): 74-79.

12. DIAS SD, et al. Tracionamento ortodôntico de canino permanente superior: relato de caso clínico. Revista Eletrônica Acervo Saúde, 2020; 41: e2418.

13. DUNCAN WR, et al. Management of nonerupted maxillary anterior tooth. J. Am. Dent. Assoc, $1983 ; 106$ (5): $640-644$.

14. ERICSON S e KUROL J. Radiographic examination of ectopically erupting maxillary canines. Am. J. Orthod. Dentofacial Orthop, 1987; 91(6): 483-492.

15. FERGUSON JW e PITT SKJ. Management of unerupted maxillary canines where no orthodontic treatment is planned; a survey of UK consultant opinion. Journal of Orthodontics, 2004; 31 (1): 28-33.

16. FILHO CL, et al., Perfuração do esmalte para o tracionamento de caninos: vantagens, desvantagens, descrição da técnica cirúrgica e biomecânica. Dental Press J Orthod, 2011;16(5): 172-205.

17. FLEMING PS, et al. Influence of radiographie position of ectopic canines on the duration of orthodontic treatment. Angle Orthodontist, 2009; 79 (3): 442-446.

18. FRANCO AA, et al. Abordagem multidisciplinar dos caninos superiores permanentes impactados. Ortodontia, 2006; 39(4): 350-9.

19. GIGLIO FPM e GURGEL JA. Abordagem cirúrgico-ortodôntica de dentes não irrompidos. Ortodontia, 2010;43(3):27986.

20. GONDIM CR et al. Prevalência de dentes retidos presentes em radiografias panorâmicas. Rev Cir Traumatol BucoMaxilo-Fac, 2010; 10(3):85-90.

21. JACOBY H. The etiology of maxillary canine impactions. Am. J. Orthod, 1983; 84 (2): 25-132.

22. JUVVADI, S. et al. Impacted canines: Etiology, diagnosis, and orthodontic management. Journal of Pharmacy and Bioallied Sciences, 2012; 4 (6): 234.

23. KANAVAKIS, G. et al. Evaluation of crown-root angulation of lateral incisors adjacent to palatally impacted canines. Progress in Orthodontics, 2015; 16 (1) 4-9.

24. LANDIM FS, et al. Avaliação clínico-radiográfica dos caninos após tratamento orto-cirúrgico, Camaragibe. Rev. Cir. Traumatol. Buco-maxilo-fac., 2010; 10(4).

25. LIMA ILA, et al. Radiografias convencionais e tomografia computadorizada cone-beam para localização de dentes inclusos: relato de caso, Arq bras odontol, 2009; 52: 58-64.

26. MANZI FR, et al. Uso da tomografia computadorizada para diagnóstico de caninos inclusos, Minas Gerais Rev. Odontol. Bras. Central, 2011; 20(53).

27. MARCELINO VCS, et al. Tratamento cirúrgico-ortodôntico do dente 33: relato de caso clínico. Archives of Health Investigation, 2017; 6(7): 304-307.

28. MARTINS MM, et al. A importância da tomografia computadorizada volumétrica no diagnóstico e planejamento ortodôntico de dentes inclusos. RGO, 2009; 57(1): 117-120.

29. PRIMO BT, et al. Dentes retidos: novas perspectivas de localização. Revista da Faculdade de Odontologia da UPF, $2011 ; 16(1)$ : 95-99.

30. PROFFIT WR. Ortodontia contemporânea. 2nd ed. Rio de Janeiro: Guanabara Koogan, 1993.

31. RIBEIRO ET, et al. Dentes inclusos associados a cistos e tumores odontogênicos: condutas terapêuticas, Vitória. Rev. Bras. Pesq. Saúde, 2015; 17(2): 78-88.

32. SILVA FILHO OG, et al. Irrupção ectópica dos caninos permanentes superiores: soluções terapêuticas. Ortodontia, 1994; 27: 50-66.

33. SILVA K, et al. Tracionamento De Caninos Inclusos: Revisão De Literatura, 2019; 31(3): 71-81.

34. SIMÃO MT, et al. Tracionamento ortodôntico de caninos superiores impactados por palatino. Revista Faipe, $2012 ; 2$ $(1)$.

35. TANAKA O, et al. O dilema dos caninos superiores impactados. Ortodontia Gaúcha, 2000; 5ç(2): 121-128.

36. TORMENA JR, et al. Caninos superiores retidos: uma reabilitação estética e funcional. J. Bras. Ortodon. Ortop Facial, 2004; 9(49):77-86.

37. VIEIRA FM, et al. Caninos impactados por palatal: correção com cantilever. Revista Amazônia Science \& Health, 2014; 2(2): 09-14.

38. WALKER L, et al. Three-dimensional localization of maxillary canines with cone-beam computed tomography. Am J Orthod Dentofacial Orthop, 2005; 128(4):418-23. 